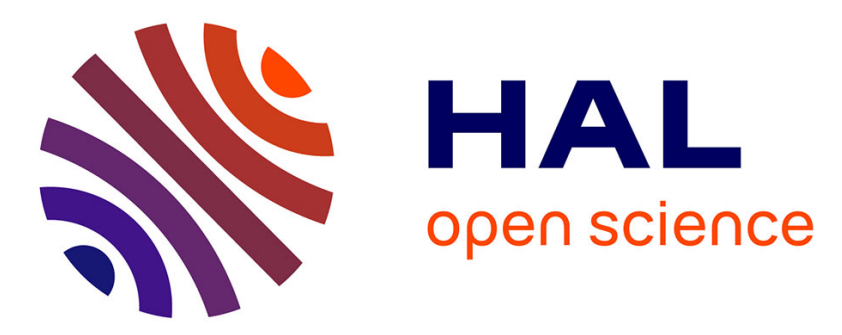

\title{
Effet d'un complexe créatine-guarana sur la puissance musculaire et la performance cognitive chez des sportifs de haut niveau de performance
}

Jeanick Brisswalter, Laura Pomportes, Karen Davranche, A. Hays, J Brisswalter Effet

\section{To cite this version:}

Jeanick Brisswalter, Laura Pomportes, Karen Davranche, A. Hays, J Brisswalter Effet. Effet d'un complexe créatine-guarana sur la puissance musculaire et la performance cognitive chez des sportifs de haut niveau de performance. Science \& Sports, 2015, 30 (4), pp.188 - 195. 10.1016/j.scispo.2015.04.002 . hal-01916078

\section{HAL Id: hal-01916078 \\ https: / hal-amu.archives-ouvertes.fr/hal-01916078}

Submitted on 8 Nov 2018

HAL is a multi-disciplinary open access archive for the deposit and dissemination of scientific research documents, whether they are published or not. The documents may come from teaching and research institutions in France or abroad, or from public or private research centers.
L'archive ouverte pluridisciplinaire HAL, est destinée au dépôt et à la diffusion de documents scientifiques de niveau recherche, publiés ou non, émanant des établissements d'enseignement et de recherche français ou étrangers, des laboratoires publics ou privés.

\section{(c)(1)}

Distributed under a Creative Commons Attribution| 4.0 International License 
archives-ouvertes

\title{
Effet d'un complexe créatine-guarana sur la puissance musculaire et la performance cognitive chez des sportifs de haut niveau de performance
}

\author{
Laura Pomportes, K. Davranche, A. Hays, J. Brisswalter
}

\section{- To cite this version:}

Laura Pomportes, K. Davranche, A. Hays, J. Brisswalter. Effet d'un complexe créatine-guarana sur la puissance musculaire et la performance cognitive chez des sportifs de haut niveau de performance. Science

Sports, 2015, 30 (4), pp.188 - 195. <10.1016/j.scispo.2015.04.002>. <hal-01916078>

\section{HAL Id: hal-01916078 \\ https://hal-amu.archives-ouvertes.fr/hal-01916078}

Submitted on 8 Nov 2018

HAL is a multi-disciplinary open access archive for the deposit and dissemination of scientific research documents, whether they are published or not. The documents may come from teaching and research institutions in France or abroad, or from public or private research centers.
L'archive ouverte pluridisciplinaire HAL, est destinée au dépôt et à la diffusion de documents scientifiques de niveau recherche, publiés ou non, émanant des établissements d'enseignement et de recherche français ou étrangers, des laboratoires publics ou privés. 
ARTICLE ORIGINAL

\title{
Effet d'un complexe créatine-guarana sur la puissance musculaire et la performance cognitive chez des sportifs de haut niveau de performance
}

\section{Effect of a creatine-guaranà complex on muscular power and cognitive performance in high-level athletes}

\author{
L. Pomportes ${ }^{a, b}$, K. Davranche ${ }^{c}$, A. Hays ${ }^{b}$, J. Brisswalter ${ }^{a, *}$ \\ a Laboratoire motricité humaine éducation sport santé, université de Nice Sophia-Antipolis, 261, \\ route de Grenoble, 06205 Nice cedex 3, France \\ ${ }^{\mathrm{b}}$ CREPS PACA, 62, chemin du Viaduc, 13080 Aix-en-Provence, France \\ c Laboratoire de psychologie cognitive (LPC), UMR 7290, Aix-Marseille université, 58, boulevard \\ Charles-Livon, 13284 Marseille, France
}

\section{MOTS CLÉS}

Créatine ;

Guarana ;

Cognition ;

Performance

musculaire

\section{Résumé}

Objectifs. - L'objectif de cette étude était de comparer les effets de la prise d'un complément à base de créatine et de guarana $(C R E+G)$ ou d'un placebo $(\mathrm{Pl})$ sur les indices de puissance musculaire et de performance cognitive chez des sportifs très entraînés.

Sujets et méthodes. - Dix-sept athlètes de haut niveau en squash et escrime ont participé à 2 sessions expérimentales présentées de façon randomisée séparées d'une semaine et correspondant à la prise d'un complément nutritionnel à base de créatine (1000 mg) et guarana $(1500 \mathrm{mg})(\mathrm{CRE}+\mathrm{G})$ ou d'un placebo $(\mathrm{Pl})$. La puissance musculaire a été évaluée lors d'un test de 6 sprints de 6 secondes avec 25 secondes de récupération réalisés sur ergocycle. La performance cognitive a été mesurée avant, et après le test de sprint par des tâches de mesure de temps de réaction simple, de vigilance, de motilité oculaire et des tâches décisionnelles (Go/No-Go). La supplémentation était présentée à l'athlète en deux prises, espacées de 30 minutes, 60 et 30 minutes avant le début de l'effort.

Résultats. - Les résultats indiquent un effet positif du complément créatine + guarana sur la puissance musculaire (fatigue et pic de puissance) ainsi que sur la performance cognitive mesurée post-exercice pour les tâches de vigilance, de Go/No-Go et de motilité oculaire. Aucun effet n'est observé sur les tâches de temps de réaction simple.

* Auteur correspondant.

Adresse e-mail : brisswalter@unice.fr (J. Brisswalter). 
Conclusion. - Le complément créatine + guarana semble présenter un effet bénéfique sur la puissance musculaire et sur la performance cognitive décisionnelle. À ce titre, ce supplément peut présenter un intérêt pour améliorer la performance sportive dans des disciplines avec une forte sollicitation des processus cognitifs pendant l'effort.

KEYWORDS
Creatine;
Guaranà;
Cognition;
Physical performance

\begin{abstract}
Summary
Background. - The aim of this study was to compare the effects of the ingestion of either a creatine + guaranà $(C R E+G)$ supplement or a placebo $(P I)$ on muscle power and cognitive performance.

Methods. - Seventeen high-level athletes specialized in squash and fencing took part in 2 randomized experimental sessions corresponding to the ingestion of a complex composed by creatine $(1000 \mathrm{mg})$ + guaranà $(1500 \mathrm{mg})$ and a placebo. The muscle power was assessed by a test of six maximal sprints on ergocycle sprints: 6 seconds long intercepted by 25 seconds of resting. Cognitive performance was assessed before and after the sprint test using a simple reaction time task, alertness task, ocular motility task and a Go/No-Go task. Supplement was ingested in a two doses, spaced from 30 minutes, 60 and 30 minutes before exercise.

Results. - Results indicate a positive effect of creatine + guaranà supplement on the muscle power (peak power and fatigue) on and cognitive performance recorded post exercise for alertness Go/No-Go and ocular motricity tasks. No effect was observed on simple reaction time.

Conclusion. - Creatine + guaranà supplement seems to have a beneficial effect on muscle power and decisional cognitive performance. This result suggests that creatine + guaranà ingestion could have an interest to enhance performance in sports with high cognitive constraints.
\end{abstract}

\section{Introduction}

La performance dans les activités physiques et sportives dépend à la fois de la capacité à produire un effort important mais aussi à la maintenir et/ou le réitérer et cela malgré l'apparition de phénomènes de fatigue. Lors de l'exercice modéré, il est communément observé une relation différente entre le niveau de sollicitation physiologique et la performance cognitive en fonction de la nature de la tâche cognitive [1]. Dans le cas d'exercice modéré de courte durée $(<\mathrm{a} 1 \mathrm{~h})$, les performances dans les tâches cognitives décisionnelles se trouvent améliorées pour une intensité modérée ou forte tandis que celles des tâches cognitives perceptuelles sont parfois altérées et ceci quelque soit l'intensité de l'exercice [2,3]. L'effet d'amélioration de la performance cognitive à l'exercice peut être expliquée par l'augmentation du flux cérébral [4] mais aussi par la modification de certains neurotransmetteurs tels que les catécholamines [2,5]. Chmura et al. (1998) [6] ont proposé d'utiliser le concept du seuil d'adrénaline pour expliquer une amélioration de la performance décisionnelle: un haut niveau d'adrénaline sanguin est associé à un changement du système nerveux central qui peut augmenter la performance cognitive.

Dans ce cadre, des travaux récents (pour revue voir, Meeusen, [7]) suggèrent que l'adoption d'une stratégie nutritionnelle particulière pourrait influencer le fonctionnement cognitif lors de l'exercice en modifiant l'état physiologique cérébral. Parmi les stratégies les plus étudiées, la prise de caféine qui est un neuro-stimulant, favorise la sécrétion de dopamine par inhibition de l'adénosine et améliore les niveaux de force, d'endurance, ou encore d'éveil $[7,8]$. En revanche, ses effets restent individuels avec parfois des effets secondaires importants (palpitations, arythmie, anxiété, nervosité...). De façon alternative, l'attention et la pratique des sportifs a récemment été portée sur la prise de guarana (Paullinia cupana) qui est une plante originaire de la forêt amazonienne utilisée depuis des milliers d'années comme stimulant par les tribus locales et que l'on retrouve depuis peu dans de nombreux compléments nutritionnels à destination des athlètes. Cette substance connue pour ses effets stimulants contient $5 \%$ à $10 \%$ de caféine mais aussi des tanins, saponines et d'autres alcaloïdes. Le guarana semble présenter au repos des effets positifs sur les tâches cognitives mais sans les effets négatifs associés à la prise de caféine [9]. En effet au repos, des études récentes ont montré que le guarana avait des effets significatifs sur des tâches de vitesse de mémorisation, les tâches décisionnelles et dans une moindre mesure sur l'altération de la vigilance avec le temps $[9,10]$. En revanche à notre connaissance aucune étude n'a étudié l'effet de la prise de guarana sur l'évolution de la performance cognitive lors de l'exercice ainsi que sur la performance musculaire.

Dans les pratiques de la population sportive, notamment à haut niveau de performance la prise de guarana est souvent associé à la créatine. Les effets de la créatine sur la performance physique sont mieux connus et il a été montré dans de nombreuses études que l'apport exogène de créatine peut augmenter la performance dans les exercices de haute intensité, notamment dans les exercices pour lesquels la phosphocréatine $(\mathrm{PCr})$ est la principale source anaérobie 
d'ATP (pour revue voir Tarnopolsky, 2010 [11]). Par ailleurs, quelques études ont également suggéré un effet positif de la créatine sur la performance cognitive $[12,13]$.

Bien que la caféine et la créatine soient des aides communément utilisées dans la performance sportive, et notamment chez les sportifs de haut niveau de performance, aucun argument réel n'existe pour justifier leur usage simultané [11]. Aussi, l'objectif principal de ce travail est d'étudier les effets conjoints de prise de créatine et de guarana sur la performance physique et cognitive. Nous émettons l'hypothèse selon laquelle l'association créatine et guarana pourrait potentialiser les effets positif de l'exercice sur la performance cognitive lors de l'exercice comparativement à un placebo.

\section{Matériel et méthodes}

\subsection{Participants}

Dix-sept sportifs de haut niveau dont 9 en escrime et 8 en squash (moyenne \pm écart-type : $18,2 \pm 3,7$ ans, 7 femmes et 10 hommes) ont participé à ce protocole. Les sujets sélectionnés ne présentaient pas de pathologie ou traitement pouvant influencer les résultats et aucun n'avait l'habitude de consommer des suppléments à base de guarana et/ou de créatine.

\subsection{Protocole expérimental}

Préalablement à la phase expérimentale, chaque sujet a été soumis à une phase d'apprentissage des tests physiques sur ergocycle WattBike ${ }^{\circledR}$ et des tests cognitifs sur ordinateur (logiciel TAP Tests d'évaluation de l'attention, version 2.3; P. Zimmermann et B. Fimm). Les critères d'apprentissage étaient l'atteinte par le sujet d'une variation inférieure ou égale à $10 \%$ par rapport au passage précédent aussi bien dans les performances cognitives que sur les valeurs de pics de puissance obtenus lors du test sur ergocyle.

La phase expérimentale était composée de deux sessions réalisées lors de deux semaines consécutives, le même jour, à la même heure pour chaque sujet et dans la même configuration d'entraînement. Lors des deux sessions expérimentales, deux conditions nutritionnelles étaient présentées au sujets dans un ordre randomisé : une condition créatine + guarana $(n=17$, groupe $C R E+G)$, et une condition placebo ( $n=17$, groupe $P()$. Le complément CRE $+G$, était un complément disponible dans le commerce pour les athlètes et se présentait sous la forme de pastilles effervescentes de créatine et d'une dose de $25 \mathrm{~mL}$ de guarana diluées dans un verre opaque de $500 \mathrm{~mL}$ Au final, le complexe CRE + G contenait $1500 \mathrm{mg}$ de guarana, $1000 \mathrm{mg}$ de créatine, $150 \mathrm{mg}$ de taurine, $133 \mathrm{mg}$ de caféine, $120 \mathrm{mg}$ de L-glutamine, $106,7 \mathrm{mg}$ de vitamine $C, 100 \mathrm{mg}$ de $L$-arginine et $1,1 \mathrm{mg}$ de vitamine B1. Le placebo était également présenté dans le même contenant opaque et était constitué d'eau gazeuse aromatisée sans sucre ajouté.

Le déroulement de chaque session expérimentale débutait par la prise de $250 \mathrm{~mL}$ du complément suivi du remplissage d'un questionnaire sur «l'état de fatigue physique et psychologique» du moment. Après 30 minutes, les sujets ingéraient les $250 \mathrm{~mL}$ restants du complément suivis de la réalisation de l'ensemble des tests cognitifs (Alerte phasique: tâche de mesure du temps de réaction simple, Balayage visuel: tâche de mesure de la vigilance, Go/No-Go: tâche de mesure des capacités d'inhibitions décisionnelles, et tâche de Motilité oculaire) au calme dans un pièce isolée (durée totale des tests cognitifs: 25 minutes). Dès la fin des tests cognitifs, les sujets réalisaient les phases d'échauffement puis un test de répétition de sprints sur ergocycle Wattbike ${ }^{\circledR}$ (durée 20 minutes). Immédiatement à l'arrêt de ce test, les sujets réalisaient dans le même espace calme deux des quatre tests cognitifs: Alerte phasique et Motilité oculaire (durée totale 15 minutes). Enfin, les sujets terminaient la session par un exercice de pédalage sous-maximal correspondant à l'intensité moyenne de compétition $(80 \%$ fréquence cardiaque maximale en escrime et $85 \%$ fréquence cardiaque maximale en squash). Pendant l'exercice, les sujets devaient réaliser les tests cognitifs des tâches de Balayage visuel et de Go/No-Go.

Les 4 tests cognitifs étaient donc réalisés 2 fois lors d'une session expérimentale : une fois intégralement avant le test de répétition de sprints (pré-exercice) puis une deuxième fois après le test physique (répétition de 6 sprints de 6 secondes sur ergocycle, post-exercice). En post-exercice, les tests Alerte phasique et Motilité oculaire étaient réalisés au repos après le test physique tandis que les tests Balayage visuel et Go/No-Go étaient réalisés pendant un exercice sous-maximal de pédalage.

\subsection{Performance musculaire}

Lors de chaque phase expérimentale, la performance musculaire a été mesurée à la suite d'un protocole d'échauffement spécifique adopté par les athlètes spécialistes d'épreuves maximales en cyclisme [14]. Le test physique consistait à réaliser une répétition de 6 sprints de 6 secondes avec 25 secondes de récupération sur ergocycle Wattbike ${ }^{\circledR}$ avec une résistance fixe selon le protocole décrit par Marquet et al. (2014) [14]. Les variables suivantes ont été analysées: le pic maximal de puissance (W) et la fatigue (\%) qui correspondaient à la différence de pic de puissance entre le meilleur des 6 sprints et le dernier.

\subsection{Performance cognitive}

\subsubsection{Temps de réaction simple}

Cette tâche renvoie à un état d'éveil général permettant de réagir rapidement et de manière appropriée aux sollicitations. Pour l'évaluer, un test de temps de réaction simple (TRS) a été réalisé dans lequel une croix apparaît au centre de l'écran avec des intervalles interstimuli variant aléatoirement, le sujet doit réagir aussi rapidement que possible en appuyant sur une touche-réponse. La moyenne des TRS a été calculée sur 20 répétitions. Un temps de réaction inférieur à $160 \mathrm{~ms}$ était considéré comme anticipé [15]. Les variables dépendantes utilisées pour ce test sont : la vitesse moyenne de réponse (mean), l'écart-type (SD) et le coefficient de variation entre les réponses d'un même bloc (CV). 


\subsubsection{Balayage visuel}

Ce test est une épreuve d'exploration de l'environnement visuel qui constitue une capacité de base de la performance attentionnelle du sujet dans une tâche d'attention focalisée. Dans cette épreuve, une matrice de $5 \times 5$ stimuli est présentée à l'écran et le sujet a pour tâche de détecter si une cible est présente dans cette matrice. Les touchesréponses sont utilisées: l'une pour signaler la présence de la cible et l'autre pour en signaler l'absence. Il s'agit d'un processus complexe dans lequel interviennent un ensemble d'éléments essentiels : intention et connaissance antérieure du sujet, sa capacité de planification, son champ visuel qui doit être intact, sa capacité à déplacer le foyer attentionnel visuel, ainsi que des mouvements oculaires efficients. Les variables dépendantes analysées pour ce test sont: la vitesse moyenne de réponse (mean), l'écart-type $(\mathrm{SD})$, le coefficient de variation entre les réponses d'un même bloc (CV), le pourcentage de fatigue (différence entre la vitesse moyenne de réponse de la fin du test et celle du début de test) ainsi que le pourcentage d'erreur (\% erreurs).

\subsubsection{Go/No-Go}

Il s'agit d'un test d'inhibition décisionnelle qui mesure la capacité à réagir de manière appropriée sous la pression du temps, tout en inhibant les réactions inadéquates.

Ici le foyer attentionnel est dirigé vers les stimuli dont l'occurrence est prévisible et qui exigent une réaction sélective, c'est-à-dire réagir, ou au contraire, s'abstenir de réagir. La forme réalisée est une forme de type « 5 stimuli dont deux cibles» : cinq formes rectangulaires dont la texture est différente apparaissent à l'écran dans un ordre pseudo-aléatoire. Deux de ces figures sont définies comme cibles et nécessitent donc d'appuyer sur la touche-réponse. Les variables dépendantes analysées pour ce test sont: la vitesse moyenne de réponse (mean), l'écart-type (SD), le coefficient de variation entre les réponses d'un même bloc (CV) ainsi que le pourcentage d'erreur.

\subsubsection{Motilité oculaire}

Il s'agit d'un test d'évaluation de la vitesse de modification de la position des yeux (regard) afin de le porter vers les informations importantes de l'environnement. Un stimulus, soit critique, soit neutre, est présenté dans un ordre pseudo-aléatoire à droite ou à gauche d'un point de fixation. Un stimulus critique apparaît également au centre de l'écran à intervalles irréguliers. Le sujet doit réagir le plus rapidement possible dès l'apparition de cette cible. Dans ce test, la latence des saccades oculaires vers la droite ou la gauche est évaluée dans une tâche de réaction. Les variables dépendantes analysées pour ce test sont: la vitesse moyenne de réponse (mean), l'écart-type (SD), le coefficient de variation entre les réponses d'un même bloc, le pourcentage de fatigue (différence entre la vitesse moyenne de réponse de la fin du test et celle du début de test) ainsi que le pourcentage d'erreur (\% erreur).

\subsection{Statistiques}

Les données recueillies lors de la phase d'apprentissage ont été recueillies puis analysées grâce au logiciel Excel ${ }^{\circledR}$ avec
Tableau 1 Valeurs de performances musculaires mesurées dans les deux conditions créatine plus guarana $(C R E+G)$ et placebo $(\mathrm{Pl})$.

\begin{tabular}{lll}
\hline & $\begin{array}{l}\text { Conditions } \\
\text { expérimentales }\end{array}$ \\
\hline & $\mathrm{Pl}$ & CRE $+\mathrm{G}$ \\
Pic de puissance & $1006,82 \pm 217,80^{\mathrm{a}}$ & $1032,76 \pm 219,13^{\mathrm{a}}$ \\
(W) & & \\
Fatigue (\%) & $-13,44 \pm 5,78^{\mathrm{a}}$ & $-10,04 \pm 3,63^{\mathrm{a}}$ \\
\hline
\end{tabular}

aLorsqu'une différence significative existe entre les conditions expérimentales.

une variation inférieure ou égale à $10 \%$ entre les deux derniers passages pour valider l'apprentissage.

Concernant la phase expérimentale, chaque variable dépendante a été analysée en utilisant un test paramétrique de Student. Le test $t$ pour échantillons appariés a été utilisé incluant les 2 conditions (créatine + guarana, $C R E+G$ et placebo, $\mathrm{Pl}$ ) à raison de 2 fois par session expérimentale. La taille de l'effet a été calculée en utilisant le $d$ de Cohen. Les données ont été analysées à l'aide du logiciel Statistica 7.1 program (StatSoft, France).

\section{Résultats}

\subsection{Performance musculaire}

L'ensemble des résultats sont présentés Tableau 1.

\subsubsection{Pic de puissance}

Une différence significative est observée entre la condition placebo $(\mathrm{Pl})$ et la condition créatine + guarana $(C R E+G)$. Dans la condition $\mathrm{Pl}$, les valeurs moyennes de puissance sont significativement inférieures à celles de la condition $C R E+G$ (respectivement pour $\mathrm{Pl}$ vs $\mathrm{CRE}+\mathrm{G}: 1006,8 \pm 217,8 \mathrm{~W}$ et $1032,7 \pm 219,1 \mathrm{~W}, p<05$, taille d'effet : $d=0,12$ ) (Tableau 1).

\subsubsection{Pourcentage Fatigue}

Pour la fatigue (diminution de la performance), une différence significative est observée entre la condition placebo $(P l)$ et la condition créatine + guarana $(C R E+G)$. Dans la condition $\mathrm{Pl}$, les valeurs moyennes de diminution de la performance (fatigue) sont significativement supérieures à celles des conditions $\mathrm{CRE}+\mathrm{G}$ (respectivement pour $\mathrm{Pl}$ vs CRE + G : $-13,44 \pm 5,78 \%$ et $-10,04 \pm 3,63 \% p<05$, taille d'effet: $d=0,73$ ) (Tableau 1).

\subsection{Performance cognitive}

L'ensemble des résultats sont présentés dans le Tableau 2.

\subsubsection{Temps de réaction simple}

Que ce soit avant ou après exercice, aucune différence significative n'est relevée entre les deux conditions Pl et CRE $+G$, $(p>, 05 ; \mathrm{NS})$ (Tableau 2). 
Tableau 2 Valeurs des performances cognitives mesurées avant ou après l'exercice dans les deux conditions créatine plus guarana $(\mathrm{CRE}+\mathrm{G})$ et placebo $(\mathrm{Pl})$.

\begin{tabular}{|c|c|c|c|c|}
\hline & \multicolumn{2}{|l|}{ Pré exercice } & \multicolumn{2}{|l|}{ Post-exercice } \\
\hline & $\mathrm{Pl}$ & $\mathrm{CRE}+\mathrm{G}$ & $\mathrm{Pl}$ & $\mathrm{CRE}+\mathrm{G}$ \\
\hline \multicolumn{5}{|l|}{ Temps de réaction simple } \\
\hline Vitesse de réponse (ms) & $222,55 \pm 35,28$ & $221,19 \pm 25,38$ & $220,38 \pm 33,60$ & $222,98 \pm 36,86$ \\
\hline Écart-type (ms) & $88,60 \pm 91,41$ & $64,94 \pm 42$ & $74,75 \pm 50,97$ & $80,02 \pm 84,48$ \\
\hline \multicolumn{5}{|l|}{ Coefficient de variance (\%) } \\
\hline \multicolumn{5}{|l|}{ Balayage visuel } \\
\hline Vitesse de réponse (ms) & $2012,35 \pm 414,36$ & $1999,44 \pm 593,04$ & $1723,63 \pm 282,51^{a}$ & $1514,13 \pm 296,51^{\circ}$ \\
\hline Écart-type (ms) & $865,91 \pm 303,13$ & $844,96 \pm 409,35$ & $681,04 \pm 193,15$ & $649,23 \pm 240,67$ \\
\hline Coefficient de variance (\%) & $42,51 \pm 8,30$ & $39,74 \pm 7,12$ & $41,46 \pm 6,78^{a}$ & $38,39 \pm 6,37^{a}$ \\
\hline Fatigue (\%) & $2,85 \pm 10,76$ & $4,13 \pm 10,49$ & $0,24 \pm 10,87$ & $1,89 \pm 9,98$ \\
\hline \multicolumn{5}{|l|}{ Erreur (\%) } \\
\hline \multicolumn{5}{|l|}{ Go/No-Go } \\
\hline Vitesse de réponse (ms) & $444,08 \pm 69,08$ & $442,16 \pm 65,06$ & $453,13 \pm 55,04^{a}$ & $424,37 \pm 50,97^{a}$ \\
\hline Écart-type (ms) & $84,22 \pm 29,63$ & $82,85 \pm 29,13$ & $88,86 \pm 36,23$ & $74,45 \pm 31,46$ \\
\hline Coefficient de variance (\%) & $18,62 \pm 4,47$ & $18,39 \pm 5,69$ & $19,25 \pm 6,14$ & $16,30 \pm 6,04$ \\
\hline \multicolumn{5}{|l|}{ Erreur (\%) } \\
\hline \multicolumn{5}{|l|}{ Motilité oculaire } \\
\hline Vitesse de réponse (ms) & $545,63 \pm 85,51^{a}$ & $519,69 \pm 68,72^{a}$ & $518,78 \pm 69,56^{a}$ & $481,62 \pm 57,30^{a}$ \\
\hline Écart-type (ms) & $134,12 \pm 67,65$ & $120,33 \pm 62,32$ & $126,19 \pm 69,75$ & $123,72 \pm 55,97$ \\
\hline Coefficient de variance (\%) & $23,84 \pm 8,64$ & $22,36 \pm 8,92$ & $23,80 \pm 9,88$ & $23,78 \pm 8,88$ \\
\hline Fatigue $(\%)$ & $0,07 \pm 7,33$ & $0,54 \pm 7,58$ & $5,16 \pm 8,19$ & $2,59 \pm 4,68$ \\
\hline Erreur (\%) & $9,27 \pm 10,12$ & $8,24 \pm 10,31$ & $7,76 \pm 10,58^{a}$ & $9,44 \pm 9,96^{a}$ \\
\hline
\end{tabular}

a'orsqu'une différence significative existe entre les conditions expérimentales.

\subsubsection{Balayage visuel}

Avant l'exercice, aucune différence significative n'est relevée entre les deux conditions Pl et CRE $+G,(p>, 05$; NS).

Après l'exercice, plusieurs différences significatives sont relevées entre les conditions expérimentales.

Une différence significative de vitesse de réponse est observée entre les condition $\mathrm{Pl}$ et $\mathrm{CRE}+\mathrm{G}$. Dans la condition $\mathrm{Pl}$, les valeurs moyennes de vitesse de réponse sont significativement supérieures à celles de la condition CRE + G (respectivement pour $\mathrm{Pl}$ vs CRE $+\mathrm{G}: 1723,63 \pm 282,51 \mathrm{~ms}$ vs 1514, $13 \pm 296,51 \mathrm{~ms} ; p<05$ taille d'effet: $d=0,72$ ).

Une différence significative du coefficient de variation est également observée entre la condition Pl et la condition $\mathrm{CRE}+\mathrm{G}$. Dans la condition Pl, les valeurs moyennes de coefficient de variation sont significativement supérieures à celles de la condition $\mathrm{CRE}+\mathrm{G}$ (respectivement pour $\mathrm{Pl}$ vs CRE + G : $41,46 \pm 6,78 \%$ vs $38,39 \pm 6,37 \% ; p<05$, taille d'effet: $d=0,50)$.

Aucune différence significative entre les conditions n'est relevée pour l'écart-type, le pourcentage de fatigue et le pourcentage d'erreur post-exercice, $(p>, 05$; NS) (Tableau 2).

\subsubsection{Go/No-Go}

Avant l'exercice, aucune différence significative n'est relevée entre les deux conditions Pl et CRE + G, $(p>, 05 ; N S)$.

Après l'exercice, une différence significative de vitesse de réponse est observée entre les conditions $P L$ et $\mathrm{CRE}+\mathrm{G}$. Dans la condition $\mathrm{Pl}$, les valeurs moyennes de vitesse de réponse sont significativement supérieures à celles de la condition $\mathrm{CRE}+\mathrm{G}$ (respectivement pour $\mathrm{Pl}$ vs CRE + G : 453, $13 \pm 55,04 \mathrm{~ms}$ vs $424,36 \pm 50,97 \mathrm{~ms} ; p<05$, taille d'effet: $d=0,55)$.

Aucune différence significative entre les conditions n'est relevée pour l'écart-type, le coefficient de variation et le pourcentage d'erreur post-exercice, $(p>, 05$; NS) (Tableau 2).

\subsubsection{Motilité oculaire}

Avant l'exercice, une différence significative de vitesse de réponse est relevée entre la condition $\mathrm{Pl}$ et la condition $\mathrm{CRE}+\mathrm{G}$. Dans la condition $\mathrm{Pl}$, les valeurs moyennes de vitesse de réponse sont significativement supérieures à celles de la condition $C R E+G$ (respectivement pour $\mathrm{Pl}$ vs CRE + G : 545,63 $\pm 85,51 \mathrm{~ms}$ vs $519,69 \pm 68,72 \mathrm{~ms} ; p<, 05$, taille d'effet: $d=0,48)$.

Aucune différence significative entre les conditions n'est relevée pour l'écart-type, le coefficient de variation, le pourcentage de fatigue et le pourcentage d'erreur préexercice.

Après l'exercice, une différence significative de vitesse de réponse est relevée entre la condition Pl et la condition $\mathrm{CRE}+\mathrm{G}$. Dans condition $\mathrm{Pl}$, les valeurs moyennes de vitesse de réponse sont significativement supérieures à celles de la condition $C R E+G$ (respectivement pour $P I$ vs $C R E+G: 518$, $78 \pm 69,56 \mathrm{~ms}$ vs $481,62 \pm 57,30 \mathrm{~ms} ; p<, 05$, taille d'effet : $d=0,58)$.

Une différence significative du pourcentage d'erreur est relevée entre la condition $\mathrm{Pl}$ et $\mathrm{CRE}+\mathrm{G}$. Dans la condition $\mathrm{Pl}$, les valeurs de pourcentage d'erreur sont significativement inférieures à celles de la condition $\mathrm{CRE}+\mathrm{G}$ (respectivement pour Pl vs CRE + G : 7,76 $\pm 10,58 \%$ et $9,44 \pm 9,96 \% ; p<, 05$, taille d'effet $d=0,21$ ). 
Aucune différence significative entre les conditions n'est relevée pour l'écart-type, le coefficient de variation et le pourcentage de fatigue mesurés post-exercice, $(p>, 05$; NS) (Tableau 2).

\section{Discussion}

Cette étude avait pour objectif d'étudier l'effet de l'ingestion d'un complément à base de créatine et de guarana comparé à un placebo sur les performances cognitives et musculaires. Les résultats principaux montrent:

- une amélioration des performances cognitives décisionnelles et de vigilance après ingestion du complément $\mathrm{CRE}+\mathrm{G}$;

- une amélioration de la puissance musculaire ainsi qu'une diminution de la fatigabilité lors de la répétition de sprints après ingestion du complexe;

- aucun effet significatif de l'ingestion du complexe CRE + G sur la performance attentionnelle simple.

La caféine et la créatine sont les composés les plus largement utilisés dans le milieu sportif. Parfois utilisés de façon simultanée, ces deux composés possèdent un effet ergogénique sur la performance physique et cognitive mais peuvent aussi présenter des effets secondaires. Les effets de la caféine sur la performance cognitive, dont le mécanisme est basé sur une action antagoniste aux récepteurs d'adénosine, ont été démontrés à plusieurs reprises. Les premières études réalisées en condition de privation de sommeil ont montré qu'une supplémentation en caféine présentait des effets bénéfiques sur la vigilance, le temps de réaction, l'éveil, la mémoire ou encore l'humeur [16]. Plusieurs études ont aussi révélé un effet bénéfique de la caféine à la fois sur la performance physique et cognitive (pour revue voir Tarnopolsky, 2010 [11]). L'ingestion de caféine permet de diminuer la douleur, la sensation perçue de l'effort, d'améliorer l'attention [8], mais aussi d'améliorer la force et l'endurance musculaire [7]. Dans une récente étude, De Pauw et al. (2015) [17] montrent également des effets bénéfiques de la caféine en rinçage de bouche sur la performance cognitive ainsi que sur l'éveil cortical. Ces derniers résultats sont très prometteurs puisque le rinçage de bouche entraîne un mécanisme central via les récepteurs de la cavité orale qui permet une activation préférentielle du cortex préfrontal, orbitofrontal et dorsolatéral [17]. Néanmoins, l'ingestion de caféine induit une grande variabilité inter-individuelles des réponses ainsi que plusieurs effets secondaires tels que: des désordres gastrointestinaux, une effet de manque pouvant engendrer des céphalées, une vision floue, des vertiges, ou d'une sensation de fatigue $[7,11,16]$.

Afin de limiter les effets secondaires liés à l'ingestion de caféine, l'attention a récemment été portée sur le guarana, utilisé comme stimulant dans de nombreux compléments nutritionnels à destination des athlètes et qui semble présenter des effets positifs sur la performance cognitive mais sans les effets secondaires associés à la caféine [9]. À notre connaissance, aucune étude ne s'est intéressée à l'effet du guarana seul sur la performance physique, en revanche, plusieurs travaux ont montré un effet bénéfique sur la cognition au repos. Lors de l'une des premières études sur l'influence du guarana sur la performance cognitive, Kennedy et al. (2007) [18] ont montré que le guarana associé à un complexe multi-vitaminé permet d'atténuer la fatigue mentale et d'augmenter la vitesse et la précision dans les tâches de Rapid Visual Information Processing (RVIP). Les tâches de RVIP, sensibles aux dysfonctions des lobes frontaux et pariétaux, mesurent l'attention soutenue ainsi que la performance générale. Dans une étude dose-réponse, Haskell et al. (2007) [10] indiquent que l'ingestion d'une dose de $75 \mathrm{mg}$ de guarana semble être optimale. Ces effets ne peuvent être attribués à la présence seule de caféine considérée comme trop faible $(9 \mathrm{mg})$ pour produire un effet actif mais plus à son interaction avec d'autres composés. Espinola et al. (1997) [19] ont montré lors d'une étude sur les rats que la seule dose de caféine contenue dans le guarana ne pouvait expliquer l'ensemble des effets trouvés sur la fonction cognitive. Il semblerait donc que les mécanismes d'action entre la caféine et le guarana soient différents et cela même si le guarana contient environ $6 \%$ à $12 \%$ de caféine. Campos et al. (2005) [20] ont montré que si le cyclopentyle adénosine, un agoniste de l'adénosine pouvait bloquer l'action de la caféine, cela n'était pas le cas avec le guarana. Les propriétés psychoactives du guarana pourraient être attribuables à plusieurs autres composés psychoactifs tels que les tannins et les saponines [19] mais aussi les vitamines et les minéraux [10]. Dans notre travail, la quantité de guarana présente dans le supplément CRE + G (1500 mg) est supérieure à celle utilisée dans de nombreuses études $(75 \mathrm{mg}$ pour Kennedy et al., 2004; $300 \mathrm{mg}$ pour Pomportes et al., 2014 et 222,2 mg pour Kennedy et al., 2007 [9,18,21]). Dans l'étude de Haskell et al. (2007) [10] sur l'effet dose-réponse, les doses de guarana les plus faibles 37,5 et $75 \mathrm{mg}$ présentent des meilleurs effets sur la performance cognitive que les doses les plus élevées (150 et $300 \mathrm{mg}$ ). L'ingestion d'une dose de $75 \mathrm{mg}$ de guarana semble produire les meilleurs effets sur la performance cognitive (Haskell et al., 2007 [10]). Dans ce cadre il est à noter que si les quantités de guarana présentes dans l'ensemble des suppléments nutritionnels sont en dessous de la quantité nécessaire pour induire un effet néfaste, plusieurs jeunes adultes ont été admis aux urgences en raison d'un overdose de caféine après ingestion de forte doses de guarana (Smith et al., 2006 [22]).

Nos résultats indiquent une amélioration des performances cognitives décisionnelles et de vigilance après ingestion de complément $C R E+G$. Ces résultats sont en accord avec les précédentes études comparant l'ingestion de guarana et d'un placebo au repos. Récemment Pomportes et al. (2014) [9], ont étudié l'effet de l'ingestion de guarana comparé à un placebo sur la variabilité cardiaque et le fonction cognitive au repos. Les résultats indiquaient un effet bénéfique de la supplémentation sur les tâches d'inhibition de la réponse de type Go/No-Go entre 30 et 90 minutes après l'ingestion mais pas d'effet sur le temps de réaction simple. Les résultats de notre étude sont similaires à ceux de cette étude puisque à la suite de l'ingestion du complexe CRE + G la performance dans la tâche de Go/No-Go est améliorée tandis que le temps de réaction simple n'est pas modifié. Il est à noter que dans notre étude, aucun effet du supplément n'est détecté au repos ce qui diffère des précédentes études. Il est possible que le test au repos ait été réalisé 
trop tôt après l'ingestion du complément puisque les tests débutaient immédiatement à la fin de la seconde prise de boisson.

Quelques études se sont intéressées à l'effet de la créatine sur la cognition mais dans des situations de stress. McMorris et al. (2007) [12], montrent un effet bénéfique de la créatine lors d'un exercice modéré sur la performance cognitive en cas de privation de sommeil. Après $24 \mathrm{~h}$ de privation de sommeil, la supplémentation en créatine induit un effet positif sur l'humeur et sur les performances cognitives complexes. De façon similaire, en condition d'hypoxie, une supplémentation en créatine améliore les capacités attentionnelles, la performance cognitive et augmente l'excitabilité corticomotrice (Turner et al., 2015 [23]). La créatine est donc également un composé psychoactif qui, en augmentant la créatine cérébrale et la phosphocréatine, permettrait d'augmenter les processus cognitifs [13].

Le second résultat important de notre étude est l'effet positif du complexe CRE + G sur la performance musculaire. Après ingestion, nos athlètes sont capables de développer un pic de puissance significativement supérieur à celui relevé dans la condition placebo $(+2,5 \%)$, (taille de l'effet: $d=0,12$, effet faible) ainsi qu'une diminution moins importante des performances au cours de la répétition de sprints $(-3,4 \%)$ (taille de l'effet: $d=0,73$, effet fort).

Ces résultats sont cohérents à ceux trouvés dans les différentes études qui ont testé l'effet de l'ingestion de Créatine sur la performance explosive (pour revue Kreider, 2003 [24]). Un apport exogène de créatine, considéré permet d'augmenter les réserves musculaires en phosphocréatine $(\mathrm{PCr})$ et en créatine et améliore la puissance explosive dans laquelle le métabolisme anaérobie est prédominant. Dans une revue de question sur le sujet, Kreider (2003) [24], indique qu'une supplémentation en créatine peut entraîner un gain de force de $15 \%$, une amélioration de la performance de 1 à $5 \%$ sur un effort de sprint isolé et de 5 à $15 \%$ sur une répétition de sprints. Bien que nos résultats soient cohérents à ceux de la littérature, notre gain de puissance reste cependant inférieur à celui reporté par Kreider. Cela peut s'expliquer par le fait que la méthode de supplémentation en créatine est généralement réalisée sur un court terme (4-5jours), voire un long terme ( 1 mois) alors que dans notre étude la supplémentation n'a été réalisée qu'en une seule dose (1000 mg), scindée en deux prises 60 et 30 minutes avant le début de l'exercice. Les mécanismes explicatifs de l'effet de la créatine seraient qu'un niveau initial en créatine phosphate augmenterait sa disponibilité et augmenterait sa vitesse de resynthèse lors des périodes de récupération. Il est à noter également que si la créatine monohydrate ne semble pas présenter pas de risque pour la santé des consommateurs, pour de fortes doses, $5 \%$ des consommateurs peuvent présenter des troubles gastrointestinaux [11].

\section{Conclusion}

L'objectif de cette étude était d'étudier les effets d'un complexe associant la créatine au guarana ( $C R E+G)$ habituellement utilisé par les athlètes sur les performances physiques et cognitives à l'effort. Nos résultats montrent que ce complexe influence favorablement la performance physique avec un effet bénéfique faible sur le pic de puissance et un effet bénéfique fort sur la fatigabilité. La performance cognitive est également positivement influencée pour les tâches de vigilance, de Go/No-Go et de motilité oculaire. En revanche, à l'inverse des études avec utilisation de la caféine, le complexe n'a pas d'effet sur le temps de réaction simple. Il sera nécessaire d'isoler les substances actives de ce complément afin de pouvoir déterminer l'impact exact de chacun sur les performances physiques et cognitives à l'effort.

\section{Déclaration d'intérêts}

Les auteurs déclarent ne pas avoir de conflits d'intérêts en relation avec cet article.

\section{Remerciement}

Ce travail a été supporté par un financement de l'Institut national du sport de l'expertise et de la performance dans le cadre de l'appel à projet en matière de performance et de sport de haut niveau.

\section{Références}

[1] Etnier JL, Chang YK. The effect of physical activity on executive function: a brief commentary on definitions, measurement issues, and the current state of the literature. $J$ sport Exerc Psychol 2009;31:469-83.

[2] Brisswalter J, Collardeau M, Rene A. Effects of acute physical exercise characteristics on cognitive performance. Sports Med 2002;32(9):555-66.

[3] Lambourne K, Tomporowski P. The effect of exercice-induced arousal on cognitive task performance: a meta-regression analysis. Brain Res 2010;1341:12-24.

[4] Chodzko-Zajko WJ. Physical fitness cognitive performance, and aging. Med Sci Sports Exerc 1991;23(7):868-72.

[5] Meeusen R, De Meirleir K. Exercice and brain neurotransmission. Sports Med 1995;20(3):160-88.

[6] Chmura J, Krysztofiak H, Ziemba AW, Nakar K, Kaciuba-Uscilko $\mathrm{H}$. Psychomotor performance during prolonged exercice above and below the blood lactate threshold. Eur J Appl Physiol 1998;77(1-2):77-80.

[7] Meeusen R. Exercice nutrition and the brain. Sports Med 2014;44(1):47-56.

[8] Glade MJ. Caffeine - not just a stimulant. Nutrition 2010;26(10):932-8.

[9] Pomportes L, Davranche K, Brisswalter I, Hays A, Brisswalter J. Heart rate variability and cognitive function following a multi-vitamin and mineral supplementation with added guarana (Paullinia cupana). Nutrients 2014;7(1):196-208.

[10] Haskell CF, Kennedy DO, Wesnes KA, Milne AL, Scholey AB. A double-blind, placebo-controlled, multi-dose evaluation of the acute behavioural effects of guaraná in humans. J Psychopharmacol 2007;21(1):65-70.

[11] Tarnopolsky MA. Caffeine and creatine use in sport. Ann Nutr Metab 2010;57(2):1-8.

[12] MCMorris T, Harris RC, Howard AN, Langridge G, Hall B, Dicks $M$, et al. Creatine supplementation, sleep deprivation, cortisol, melatonin and behavior. Physiol Behav 2007;90:21-8.

[13] Rawson ES, Venezia AC. Use of creatine in the elderly and evidence for effects on cognitive function in young and old. Amino Acids 2011;40(5):1349-62. 
[14] Marquet LA, Hausswirth C, Hays A, Vettoretti F, Brisswalter J. Comparison of between-training-sessions recovery strategies for world-class BMX pilots. Int J Sports Physiol Perform 2014;10(2):219-23.

[15] Collardeau M, Brisswalter J, Audiffren M. Effects of a prolonged run on simple reaction time of well-trained runners. Percept Mot Skills 2001;93(3):679-89.

[16] Lieberman HR, Tharion WJ, Shukitt-Hale B, Speckman KL, Tulley R. Effects of caffeine, sleep loss, and stress on cognitive performance and mood during U.S. Navy SEAL training. Psychopharmacology 2002;164(3):250-61.

[17] De Pauw K, Roelands B, Knaepen K, Polfliet M, Stiens J, Meeusen R. Effects of caffeine and maltodextrin mouth rinsing on P300, brain imaging, and cognitive performance. $J$ Appl Physiol 2015;118(6):776-82, http://dx.doi.org/10.1152/ japplphysiol.01050.2014.

[18] Kennedy DO, Haskell CF, Robertson B, Reay J, Brewster-Maund C, Luedemann J, et al. Improved cognitive performance and mental fatigue following a multi-vitamin and mineral supplement with added guaraná (Paullinia cupana). Appetite 2007;50:506-13.
[19] Espinola EB, Dias RF, Mattei R, Carlini EA. Pharmacological activity of guarana (Paullinia Cupana Mart.) in laboratory animals. J Ethnopharmacol 1997;55(3):223-9.

[20] Campos AR, Barros Al, Albuquerque FA, Leal LK, Rao VS. Acute effects of guarana (Paullinia cupana Mart.) on mouse behaviour in forced swimming and open field tests. Phytother Res 2005;19(5):441-3.

[21] Kennedy DO, Haskell CF, Wesnes KA, Scholey AB. Improved cognitive performance in human volunteers following administration of guarana (Paullinia cupana) extract: comparison and interaction with Panax ginseng. Pharmacol Biochem Behav 2004;79:401-11.

[22] Smith AP, Christopher G, Sutherland D. Effects of caffeine in overnight-withdrawn consumers and non-consumers. Nutr Neurosci 2006;9(1-2):63-71.

[23] Turner CE, Byblow WD, Gant N. Creatine supplementation enhances corticomotor excitability and cognitive performance during oxygen deprivation. J Neurosci 2015;35(4):1773-80.

[24] Kreider RB. Effects of creatine supplementation on performance and training adaptations. Mol Cell Biochem 2003;244:89-94. 